\title{
Detection and quantification of cellulolytic bacteria in the equine caecum using oligonucleotide probes targeting 16S rRNA
}

\author{
L Millet 1, V Julliand 2, G Fonty 1 \\ 1 INRA, Laboratoire de Microbiologie, C.R. de Clermont-Ferrand-Theix, 63122 Saint-Genès-Champanelle \\ 2 Laboratoire associé de Recherches Zootechniques, INRA, ENESAD, 26 Boulevard du Docteur \\ Petit Jean, 21000 Dijon, France
}

Identification and enumeration of microbial populations are central to studies of microbial ecology. The microbial community structure of rumen and gut ecosystems has been intensively studied using traditional microbiological techniques. However, probably because of the limitations and problem associated with these culture-based methods the structure of the bacterial cellulolytic population of the hundgut of herbivorous hingut-fermenters such as equines is still almost unknown. The recent development of phylogenetically-based hybrization probes offers a powerful and rapid technique for studying the ecology of intestinal ecosystems. The purpose of this work was the use of $16 \mathrm{~S}$ rRNA-targeted oligonucleotide probes to detect and quantify the cellulolytic bacteria in the caecum of poneys and asses.

Three poneys and 3 asses fed a roughagediet were used in this study. Cecal samples were collected through a canula fitted on the caecum, frozen at $-80^{\circ} \mathrm{C}$ and then freeze-dried. Extraction of RNA and labeling of probes were performed according to the techniques described by Stahl et al (1988, Appl Environ Microbiol, 1079-1084). Four 32P-labelled probes, complementary to regions of the small subunit rRNA were used: the universal probe EUB 338 targeted the total bacterial rRNA
(Stahl and Amann, 1991, in: Sequencing and hybridization techniques in bacterial systematics, Stakebrandt and Goodfellow, eds, 205248), probe F650 targeted Fibrobacter succinogenes (Amann et al, 1990, J Bacteriol, 172, 762-770). Probes RAL 196 and RFL 1269 targeted Ruminococcus albus and $R$. flavefaciens respectively (Odenyo et al, 1994, Appl Environ Microbiol, 60, 3688-3696). The proportion of each cellulolytic species was expressed as a percentage of total bacterial 16S rRNA.

R. flavefaciens was the dominant species in caecal contents of both of poneys and asseses. However, its population represented only between 0.2 and 1 percent of the total cecal $16 S$ rRNA. The population of $F$. succinogenes was very low. Except in one poney and one ass its proportion was less than 0.1 percent of total 16S rRNA. $R$. albus was only detected as traces. Quantitatively these results are in agreement with the numeration of cellulolytic bacteria in the caecum of equines usually obtained with culture-based techniques. R. flavefaciens has also been found as the dominant species in poneys and asses using the isolation procedure and culture-based description (Julliand, unpublished data). 